Pacific Journal of Mathematics

CHARACTERIZING LOCAL CONNECTEDNESS IN INVERSE 


\title{
CHARACTERIZING LOCAL CONNECTEDNESS IN INVERSE LIMITS
}

\author{
G. R. Gordh, JR. ANd Sibe Mardešić
}

Let $X$ denote the limit of an inverse system $\underline{X}=$ $\left\{X_{\alpha} ; p_{\alpha \alpha^{\prime}} ; A\right\}$ of locally connected Hausdorff continua. The main purpose of this paper is to define a notion of local connectedness for inverse systems, and to prove that if $\underline{X}$ is locally connected, then so is the limit $X$. If the bonding maps $p_{\alpha \alpha^{\prime}}$ are surjections, then $X$ is locally connected if and only if $\underset{X}{ }$ is. The following corollaries are obtained. (1) If $\underline{X}$ is $\sigma$-directed and surjective, then $X$ is locally connected. (2) If $\underline{X}$ is well-ordered, surjective, and weight $\left(X_{\alpha}\right) \leqq \lambda$ for each $\alpha$ in $\bar{A}$, then either weight $(X) \leqq \lambda$, or $X$ is locally connected. (3) If $X$ is $\sigma$-directed and the factor spaces $X_{\alpha}$ are trees (generalized arcs), then $X$ is a tree (generalized arc). (4) If $\underline{X}$ is well-ordered and the factor spaces $X_{\alpha}$ are dendrites (arcs), then either $X$ is metrizable, or $X$ is a tree (generalized arc).

1. Introduction. By a continuum we mean a compact connected Hausdorff space. Let $X$ denote the limit of an inverse system $\underline{X}=\left\{X_{\alpha} ; p_{\alpha \alpha^{\prime}} ; A\right\}$ where the factor spaces $X_{\alpha}$ are locally connected continua, and $A$ is an arbitrary directed set. It is well-known that every continuum $X$ can be obtained as the limit of such a system where the factor spaces are polyhedra (see Theorem 10.1, p. 284, [2]). Hence local connectedness of the factor spaces $X_{\alpha}$ does not imply local connectedness of the limit $X$. It is the main purpose of this paper to introduce a notion of local connectedness for inverse systems, and to prove that for such systems $\underline{X}$ the limit space $X$ is locally connected (see Theorem 1 ). The converse holds if $\underline{X}$ is a surjective system, i.e., if the bonding maps $p_{\alpha \alpha^{\prime}}$ are surjections. An immediate corollary is the known result that if $\underline{X}$ is a monotone inverse system, then $X$ is locally connected [1].

In $\$ 3$ the main theorem is applied to well-ordered and $\sigma$-directed inverse systems, i.e., systems in which every countable subset of the index set is bounded above. The following somewhat surprising results are obtained. (1) If the inverse system $\underset{X}{ }$ is $\sigma$-directed and surjective, then the limit $X$ is locally connected. (2) If $\underline{X}$ is well-ordered, surjective, and weight $\left(X_{\alpha}\right) \leqq \lambda$ for each $\alpha$ in $A$, then weight $(X) \leqq \lambda$ or $X$ is locally connected.

Section 4 contains similar results about well-ordered and $\sigma$ directed inverse systems of trees (i.e., locally connected, hereditarily unicoherent continua [9]) and generalized arcs (i.e., ordered continua). 
For example, the limit of a $\sigma$-directed inverse system of trees (generalized arcs) is a tree (generalized arc). ${ }^{1}$

The problem of characterizing locally connected inverse limits has been studied from a different point of view in [3].

The reader is referred to [1] for basic results concerning inverse limits of compact Hausdorff spaces.

2. Locally connected inverse systems. A continuum $X$ has property $S$ if given any open cover $U$ of $X$, there exists a finite cover $\mathscr{C}$ of $X$ which refines $\mathcal{U}$ and consists of connected subsets of $X$. A continuum is locally connected if and only if it has property $S$ (e.g., Chapter IV, Theorem 3.7, p. 106, [11]).

Definition Let $f: X \rightarrow Y$ be a mapping of locally connected continua, and let $F \subset U \subset Y$ where $F$ is closed and $U$ is open. We define the splitting number $s(f, U, F)$ of the triple $(f, U, F)$ to be the number of components of $f^{-1}(U)$ which meet $f^{-1}(F)$.

\section{Lemma 1. The splitting number $s(f, U, F)$ is finite.}

Proof. Since $X$ is locally connected, the components of $f^{-1}(U)$ are open sets. By compactness, only finitely many components of $f^{-1}(U)$ can meet the closed set $f^{-1}(F)$.

Definition. Let $\underset{X}{X}=\left\{X_{\alpha} ; p_{\alpha \alpha}{ }^{\prime} ; A\right\}$ be an inverse system of continua over an arbitrary directed set $A$. We say that the system $\underline{X}$ is locally connected if (1) the factor spaces $X_{\alpha}$ are locally connected; and (2) whenever $F_{\alpha} \subset U_{\alpha} \subset X_{\alpha}$, where $F_{\alpha}$ is closed and $U_{\alpha}$ is open, there exists an $\alpha^{\prime} \geqq \alpha$ in $A$ such that the splitting number $s\left(p_{\alpha \alpha^{\prime}}, U_{\alpha}, F_{\alpha}\right)$ agrees with $s\left(p_{\alpha \alpha^{\prime \prime}}, U_{\alpha}, F_{\alpha}\right)$ for every $\alpha^{\prime \prime} \geqq \alpha^{\prime}$.

THEOREM. 1. The limit of a locally connected inverse system is locally connected.

Proof. Let $\underline{X}=\left\{X_{\alpha} ; p_{\alpha \alpha^{\prime}} ; A\right\}$ be a locally connected inverse system with limit $X$ and projections $p_{\alpha}: X \rightarrow X_{\alpha}$. We shall prove that $X$ has property $S$. Let $U$ be any open cover of $X$. There exists an $\alpha \in A$ and a finite open cover $\mathcal{U}_{\alpha}=\left(U_{1}, \cdots, U_{n}\right)$ of $X_{\alpha}$ such that $\left\{p_{\alpha}^{-1}\left(U_{i}\right)\right\}_{i=1}^{n}$ refines $U$ (e.g., Lemma 3.7, p. 263, [2]). Choose open covers $U_{\alpha}^{\prime}=$ $\left(U_{1}^{\prime}, \cdots, U_{n}^{\prime}\right)$ and $U_{\alpha}^{\prime \prime}=\left(U_{1}^{\prime \prime}, \cdots, U_{n}^{\prime \prime}\right)$ of $X_{\alpha}$ such that $U_{i}^{\prime \prime} \subset \operatorname{cl}\left(U_{i}^{\prime \prime}\right) \subset U_{i}^{\prime} \subset$ $\operatorname{cl}\left(U_{i}^{\prime}\right) \subset U_{i}$. Let $F_{i}=\operatorname{cl}\left(U_{i}^{\prime \prime}\right)$ and consider the pairs $\left(U_{i}^{\prime}, F_{i}\right)$. Since the system $X$ is locally connected, there exists an $\alpha^{\prime} \in A$ such that for $\alpha^{\prime \prime} \geqq \alpha^{\prime}$ we have $s\left(p_{\alpha \alpha^{\prime}}, U_{i}^{\prime}, F_{i}\right)=s\left(p_{\alpha \alpha^{\prime \prime}}, U_{i}^{\prime}, F_{i}\right)$ for $1 \leqq i \leqq n$. Let $s_{i}$ denote the splitting number $s\left(p_{\alpha \alpha^{\prime}}, U_{i}^{\prime}, F_{i}\right)$. For $\alpha^{\prime} \in A$ as above, let

M. Smith has announced results similar to Corollary 5 and Theorem 6 at the Topology Conference held at the University of North Carolina at Charlotte, March, 1974. 
$\left\{V_{a^{\prime} j}^{i}\right\}_{j=1}^{s_{i}}$ denote the collection of components of $p_{\alpha \alpha^{\prime}}{ }^{-1}\left(U_{i}^{\prime}\right)$ which intersect $p_{\alpha \alpha^{\prime}}{ }^{-1}\left(F_{i}\right)$. For $\alpha^{\prime \prime} \geqq \alpha^{\prime}$ there are also $s_{i}$ components of $p_{\alpha \alpha^{\prime}}{ }^{-1}\left(U_{i}^{\prime}\right)$ which intersect $p_{\alpha \alpha^{\prime}}{ }^{-1}\left(F_{i}\right)$. Denote these components by $\left\{V_{\alpha^{\prime \prime} j}^{i}\right\}_{j=1}^{s_{i}}$, and assume that they are labelled so that $p_{\alpha^{\prime} \alpha^{\prime \prime}}\left(V_{\alpha^{\prime \prime} j}^{i}\right) \subset V_{\alpha^{\prime} j}^{i}$. Define $C_{\alpha^{\prime \prime j}}^{i}=$ $\operatorname{cl}\left(V_{\alpha^{\prime \prime} j}^{i}\right)$ for all $\alpha^{\prime \prime} \geqq \alpha^{\prime}$, and let

$$
C_{j}^{i}=\operatorname{inv} \lim \left\{C_{\alpha^{\prime \prime} j}^{i} ; \alpha^{\prime \prime} \geqq \alpha^{\prime}\right\}
$$

Since $\left\{F_{i}\right\}$ covers $X_{\alpha}$, it follows that $\left\{C_{\alpha^{\prime j}}^{i}\right\}$ covers $X_{\alpha^{\prime \prime}}$ for each $\alpha^{\prime \prime} \geqq \alpha^{\prime}$. To every $x \in X$ one can assign a pair $(i, j)$ such that $p_{\alpha^{\prime \prime}}(x) \in C_{\alpha^{\prime \prime j} \text {. Since } i}^{i}$ and $j$ vary through a finite set, some pair $(i, j)$ occurs cofinally often; and consequently $x \in C_{j}^{i}$. Consequently, $\left\{C_{j}^{i}\right\}_{i, j}$ covers $X$ and refines $\left\{p_{\alpha}^{-1}\left(U_{i}\right)\right\}_{i=1}^{n}$ which refines $\mathcal{U}$. Since each $C_{i}^{i}$ is a subcontinuum of $X$, it follows that $X$ has property $S$.

The next theorem provides a converse to Theorem 1 for inverse systems with surjective bounding maps.

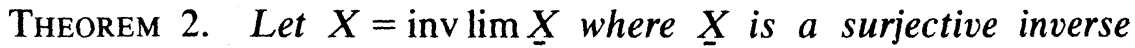
system of continua. If $X$ is locally connected, then the system $X$ is locally connected.

The proof of Theorem 2 depends on two simple lemmas.

Lemma 2. Let $X_{1}, X_{2}$ and $Y$ be locally connected continua and suppose that $f_{i}: X_{i} \rightarrow Y(i=1,2)$ and $g: X_{2} \rightarrow X_{1}$ are continuous surjec tions such that $f_{2}=f_{1} g$. Let $F \subset U \subset Y$ where $F$ is closed and $U$ is open. Then $s\left(f_{1}, U, f\right) \leqq s\left(f_{2}, U, F\right)$.

Proof. Let $s_{1}=s\left(f_{1}, U, F\right)$, and let $V_{1}, \cdots, V_{s_{1}}$ denoted the components of $f_{1}^{-1}(U)$ which meet $f_{1}^{-1}(F)$. For each $i \leqq s_{1}$, at least one component of $g^{-1}\left(V_{i}\right)$ meets $g^{-1}\left(f_{1}^{-1}(F)\right)=f_{2}^{-1}(F)$. Since each component of $g^{-1}\left(V_{i}\right)$ is a component of $f_{2}^{-1}(U)$, at least $s_{1}$ components of $f_{2}^{-1}(U)$ meet $f_{2}^{-1}(F)$. Thus $s_{1} \leqq s\left(f_{2}, U, F\right)$.

Lemma 3. Let $A$ be a directed set and $N$ the set of natural numbers. If $\pi: A \rightarrow N$ is an order preserving bounded function, then $\pi$ is eventually constant.

Proof. Let $m=\max \pi(A)$, and choose $\alpha \in A$ such that $\pi(\alpha)=$ $m$. Thus for $\alpha^{\prime} \geqq \alpha, \pi\left(\alpha^{\prime}\right)=m$.

Proof of Theorem 2. Let $\underset{X}{X}=\left\{X_{\alpha} ; p_{\alpha \alpha^{\prime}} ; A\right\}$ be a surjective system of continua with locally connected limit $X$ and projections $p_{\alpha}: X \rightarrow X_{\alpha}$. Since the projections $p_{\alpha}$ are surjections (e.g., Theorem 2.6, [1]), each 
factor space $X_{\alpha}$ is the image of a locally connected continuum; hence each $X_{\alpha}$ is locally connected (e.g., Theorem 3-22, p. 126, [5]). Given $\alpha \in A$, let $A(\alpha)=\left\{\alpha^{\prime} \in A \mid \alpha^{\prime} \geqq \alpha\right\}$, and let $F_{\alpha} \subset U_{\alpha} \subset X_{\alpha}$ where $F_{\alpha}$ is closed and $U_{\alpha}$ is open. Define $\pi: A(\alpha) \rightarrow N$ by $\pi\left(\alpha^{\prime}\right)=s\left(p_{\alpha \alpha^{\prime}}, U_{\alpha}, F_{\alpha}\right)$. Lemma 2 implies that $\pi$ is order preserving and bounded by $s\left(p_{\alpha}, U_{\alpha}, F_{\alpha}\right)$. By Lemma 3, there exists $\alpha^{\prime} \in A(\alpha)$ such that for all $\alpha^{\prime \prime} \geqq \alpha^{\prime}, \pi\left(\alpha^{\prime}\right)=\pi\left(\alpha^{\prime \prime}\right)$; i.e., $s\left(p_{\alpha \alpha^{\prime}}, U_{\alpha}, F_{\alpha}\right)=s\left(p_{\alpha \alpha^{\prime \prime}}, U_{\alpha}, F_{\alpha}\right)$.

Corollary 1. Let $X$ be a surjective inverse system of locally connected continua with limit $X$. Then $X$ is locally connected if and only if $\underline{X}$ is locally connected.

A surjective continuous function $f: X \rightarrow Y$ between continua is monotone if $f^{-1}(y)$ is a continuum for each $y \in Y$. An inverse system of continua is monotone if each bonding map is monotone.

COROllary 2. (Capel [1]). The limit of a monotone inverse sys tem of locally connected continua is locally connected.

Proof. Let $\left\{X_{\alpha} ; p_{\alpha \alpha^{\prime}} ; A\right\}$ be a monotone inverse system of locally connected continua. Let $F_{\alpha} \subset U_{\alpha} \subset X_{\alpha}$ where $F_{\alpha}$ is closed and $U_{\alpha}$ is open in $X_{\alpha}$. If $\alpha^{\prime} \geqq \alpha$, then since $p_{\alpha \alpha^{\prime}}$ is monotone, the splitting number $s\left(p_{\alpha \alpha^{\prime}}, U_{\alpha}, F_{\alpha}\right)$ is precisely the number of components of $U_{\alpha}$ which meet $F_{\alpha}$. Thus, for $\alpha^{\prime} \geqq \alpha$ the splitting number $s\left(p_{\alpha \alpha^{\prime}}, U_{\alpha}, F_{\alpha}\right)$ is independent of $\alpha^{\prime}$, and so the inverse system is locally connected. By Theorem 1, the limit of the system is locally connected.

3. Well-ordered and $\sigma$-directed inverse systems of locally connected continua. We say that a quasi-ordered set $\boldsymbol{A}$ is $\sigma$-directed (directed) if every countable (finite) subset of $A$ is bounded above. Thus every bounded quasi-ordered set is $\sigma$-directed. Clearly, an unbounded well-ordered set is $\sigma$-directed if and only if it contains no cofinal sequence. Another example of a $\sigma$-directed set is the collection of all countable subsets of a given set, ordered by inclusion. An inverse system is said to be $\sigma$-directed (well-ordered) if its index set is $\sigma$-directed (well-ordered).

Lemma 4. Let $A$ be a $\sigma$-directed set and let $N$ denote the set of naturai numbers. If $\pi: A \rightarrow N$ is an order preserving function, then $\pi$ is eventually constant.

Proof. If $\pi$ is not eventually constant, then there exists an increasing sequence $\left\{\alpha_{i}\right\}_{i=1}$ in $A$ such that $\left\{\pi\left(\alpha_{i}\right)\right\}_{i=1}$ is cofinal in $N$. 
Since $A$ is $\sigma$-directed, there exists $\alpha \in A$ such that $\alpha_{i} \leqq \alpha$ for every $i \in N$. Thus $\pi\left(\alpha_{i}\right) \leqq \pi(\alpha)$ for every $i$, which is a contradiction.

THEOREM 3. The limit of a $\sigma$-directed surjective inverse system of locally connected continua is locally connected.

Proof. Let $\underset{X}{X}=\left\{X_{\alpha} ; p_{\alpha \alpha^{\prime}} ; A\right\}$ be a $\sigma$-directed surjective inverse system of locally connected continua. According to Theorem 1 , it suffices to show that $\underline{X}$ is a locally connected system. Let $F_{\alpha} \subset U_{\alpha} \subset X_{\alpha}$ where $F_{\alpha}$ is closed and $U_{\alpha}$ is open. Let $A(\alpha)=\left\{\alpha^{\prime} \in A \mid \alpha^{\prime} \geqq \alpha\right\}$ and note that $A(\alpha)$ is a $\sigma$-directed set. We define a function $\pi: A(\alpha) \rightarrow N$ by $\pi\left(\alpha^{\prime}\right)=s\left(p_{\alpha \alpha^{\prime}}, U_{\alpha}, F_{\alpha}\right)$. By Lemma $2, \pi$ is an increasing function. Thus, by Lemma $4, \pi$ is eventually constant, and there exists $\alpha^{\prime} \in A(\alpha)$ such that $\pi\left(\alpha^{\prime}\right)=\pi\left(\alpha^{\prime \prime}\right)$ whenever $\alpha^{\prime} \leqq \alpha^{\prime \prime}$. Thus for $\alpha^{\prime} \leqq \alpha^{\prime \prime}$ we have $s\left(p_{\alpha \alpha^{\prime}}, U_{\alpha}, F_{\alpha}\right)=s\left(p_{\alpha \alpha^{\prime \prime}}, U_{\alpha}, F_{\alpha}\right)$, and $\underline{X}$ is locally connected.

COROllary 3. If $X$ is the limit of a $\sigma$-directed inverse system of hereditarily locally connected continua, then $X$ is hereditarily locally connected.

Proof. Let $X=$ inv $\lim \left\{X_{\alpha} ; p_{\alpha \alpha^{\prime}} ; A\right\}$ where $A$ is $\sigma$-directed and the factor spaces $X_{\alpha}$ are hereditarily locally connected continua. Let $Y$ be any subcontinuum of $X$. Then $\left\{p_{\alpha}(Y) ; p_{\alpha \alpha^{\prime}} \mid p_{\alpha^{\prime}}(Y) ; A\right\}$ is a $\sigma$-directed surjective inverse system of locally connected continua with limit $Y$ (see [1]). By Theorem 3, Y is locally connected.

The weight of a topological space $X$, denoted $w(X)$, is the smallest cardinal number $\lambda$ such that $X$ admits a basis for its topology of cardinality $\cdot \lambda$.

THEOREM 4. Let $X$ be the limit of a well-ordered surjective inverse system $X$ of locally connected continua $X_{\alpha}$ such that $w\left(X_{\alpha}\right) \leqq \lambda$ for each $X_{\alpha}$. Then, either $w(X) \leqq \lambda$, or $X$ is locally connected. In particular, if the factor spaces $X_{\alpha}$ are metrizable, then either $X$ is metrizable, or $X$ is locally connected.

Proof. Let $A$ denote the well-ordered index set for the system $X$. If $A$ contains a cofinal sequence, then $X$ is the limit of an inverse sequence of continua $X_{n}$ such that $w\left(X_{n}\right) \leqq \lambda$; hence $w(X) \leqq$ $\lambda$. Otherwise, $A$ is $\sigma$-directed and $X$ is locally connected by Theorem 3. 
Remark. Suppose that the nonmetrizable continuum $X$ is the limit of a well-ordered surjective inverse system of metric continua $X_{\alpha}$. If $X$ is non-locally connected, then by Theorem 4 the factor spaces $X_{\alpha}$ are eventually nonlocally connected as well. This remark applies to all continua of weight $\kappa_{1}$, since such continua are known to be limits of well-ordered surjective inverse systems of metric continua [7].

COROllary 4. Let $X$ be the limit of a well-ordered inverse system $\underline{X}$ of hereditarily locally connected continua $X_{\alpha}$ such that $w\left(X_{\alpha}\right) \leqq \lambda$ for each $\alpha \in A$. Then either $w(X) \leqq \lambda$, or $X$ is hereditarily locally connected.

4. Well-ordered and $\sigma$-directed inverse systems of trees and generalized arcs. A continuum $X$ is a tree [9] if each pair of points is separated by a third point. A continuum $X$ with precisely two nonseparating points is called a generalized arc (or an ordered continuum). According to [9], a continuum $X$ is a tree if and only if $X$ is locally connected and hereditarily unicoherent. Clearly every subcontinuum of a tree $X$ is a tree, and consequently $X$ is hereditarily locally connected. It follows immediately from Theorem 4.1(3) of [4] that a tree is a generalized arc if and only if it is atriodic.

It is known that the limit of a monotone inverse system of trees is a tree (see the proof of Theorem 4.2 in [4]); and that the limit of a monotone inverse system of generalized arcs is a generalized arc (Lemma 4.7 of [1], or [8]). We shall obtain the same conclusions for $\sigma$-directed inverse systems of trees and generalized arcs without any assumptions about the bonding maps.

Lemma 5. Suppose that $X$ is the limit of an arbitrary inverse system of trees (generalized arcs). If $X$ is locally connected, then $X$ is a tree (generalized arc).

Proof. Since the factor spaces are hereditarily unicoherent, $X$ is also hereditarily unicoherent by a routine application of ((2.9), p. 235, [1]). Consequently, $X$ is a tree. If the factor spaces are generalized arcs, then $X$ is chainable (e.g., [6]). Since chainable continua are atriodic, $X$ is an atriodic tree; i.e., a generalized arc.

REMARK. The proof of Lemma 5 can be modified to show that a locally connected tree-like (arc-like, i.e., chainable) continuum is a tree (generalized arc). If $X$ is tree-like, then $X$ is hereditarily unicoherent. Consequently, if $X$ is locally. connected, then $X$ is a tree. If, in addition, $X$ is arc-like, then $X$ is atriodic; hence $X$ is a generalized arc (see [8] for a different proof). 
THEOREM 5. If $X$ is the limit of a $\sigma$-directed inverse system of trees (generalized arcs), then $X$ is a tree (generalized arc).

Proof. Apply Corollary 3 and Lemma 5.

THEOREM 6. Let $X$ be the limit of a well-ordered inverse system of trees (generalized arcs) $X_{\alpha}$ such that $w\left(X_{\alpha}\right) \leqq \lambda$ for each $X_{\alpha}$. Then, either $w(X) \leqq \lambda$, or $X$ is a tree (generalized arc).

Proof. Apply Corollary 4 and Lemma 5.

COROLlary 5. Let $X$ be the limit of a well-ordered inverse system of dendrites (arcs). Then, either $X$ is metrizable, or $X$ is a tree (generalized arc).

Proof. A dendrite $(\operatorname{arc})$ is a metrizable tree (generalized $\operatorname{arc}$ ) (see (1.1), p. 88 and Theorem (6.2), p. 54 of [10]). Thus the desired conclusion follows from Theorem 6.

REMARK. The limit of a well-ordered inverse system of arcs need not be metrizable. For example, the long line (p. 55, [5]) is the limit of a well-ordered monotone inverse system of arcs.

\section{REFERENCES}

1. C. E. Capel, Inverse limit spaces, Duke Math. J., 21 (1954), 233-246.

2. S. Eilenberg and N. Steenrod, Foundations of algebraic topology, Princeton Univ. Press, Princeton, N.J., 1952.

3. M. K. Fort, Jr. and J. Segal, Local connectedness of inverse limit spaces, Duke Math. J., 28 (1961), 253-260.

4. G. R. Gordh, Jr., Monotone decompositions of irreducible Hausdorff continua, Pacific J. Math., 36 (1971), 647-658.

5. J. G. Hocking and G. S. Young, Topology, Addison-Wesley, Reading, Mass., 1961.

6. S. Mardešic, Chainable continua and inverse limits, Glasnik Mat. Fiz. Astr., 14 (1959), 219-232.

7. - On covering dimension and inverse limits of compact spaces, Illinois J. Math., 4 (1960), 278-291.

8. Locally connected, ordered and chainable continua, Rad Jugoslav. Akad. Znan. Umjetn., 319 (1960), 147-166.

9. L. E. Ward, Jr., Mobs, trees and fixed points, Proc. Amer. Math. Soc., 8 (1957), 798-804.

10. G. T. Whyburn, Analytic topology, Amer. Math. Soc. Colloquium Publications 28, Providence, 1942.

11. R. L. Wilder, Topology of manifolds, Amer. Math. Soc. Colloquium Publications 32, Providence, 1949.

Received February 25, 1974. During this research the first author was visiting the University of Zagreb on an exchange program sponsored jointly by the National Academy of Sciences (U.S.A.) and the Yugoslav Academy of Sciences and Arts. 



\section{PACIFIC JOURNAL OF MATHEMATICS}

\section{EDITORS}

RICHARD ARENS (Managing Editor)

University of California

Los Angeles, California 90024

\section{J. DugundJI}

Department of Mathematics University of Southern California Los Angeles, California 90007

D. Gilbarg and J. Milgram

Stanford University

Stanford, California 94305

\section{ASSOCIATE EDITORS}
E. F. BECKENBACH
B. H. NeumanN
F. WoLF
K. YoshiDA

\section{SUPPORTING INSTITUTIONS}

\author{
UNIVERSITY OF BRITISH COLUMBIA \\ CALIFORNIA INSTITUTE OF TECHNOLOGY \\ UNIVERSITY OF CALIFORNIA \\ MONTANA STATE UNIVERSITY \\ UNIVERSITY OF NEVADA \\ NEW MEXICO STATE UNIVERSITY \\ OREGON STATE UNIVERSITY \\ UNIVERSITY OF OREGON \\ OSAKA UNIVERSITY
}

\author{
UNIVERSITY OF SOUTHERN CALIFORNIA \\ STANFORD UNIVERSITY \\ UNIVERSITY OF TOKYO \\ UNIVERSITY OF UTAH \\ WASHINGTON STATE UNIVERSITY \\ UNIVERSITY OF WASHINGTON \\ AMERICAN MATHEMATICAL SOCIETY
}

The Supporting Institutions listed above contribute to the cost of publication of this Journal, but they are not owners or publishers and have no responsibility for its contents or policies.

Mathematical papers intended for publication in the Pacific Journal of Mathematics should be in typed form or offset-reproduced (not dittoed), double spaced with large margins. Underline Greek letters in red, German in green, and script in blue. The first paragraph or two must be capable of being used separately as a synopsis of the entire paper. Items of the bibliography should not be cited there unless absolutely necessary, in which case they must be identified by author and Journal, rather than by item number. Manuscripts, in duplicate, may be sent to any one of the four editors. Please classify according to the scheme of Math. Reviews, Index to Vol. 39. All other communications should be addressed to the managing editor, or Elaine Barth, University of California, Los Angeles, California, 90024.

100 reprints are provided free for each article, only if page charges have been substantially paid. Additional copies may be obtained at cost in multiples of 50 .

The Pacific Journal of Mathematics is issued monthly as of January 1966. Regular subscription rate: $\$ 72.00$ a year (6 Vols., 12 issues). Special rate: $\$ 36.00$ a year to individual members of supporting institutions.

Subscriptions, orders for back numbers, and changes of address should be sent to Pacific Journal of Mathematics, 103 Highland Boulevard, Berkeley, California, 94708.

PUBLISHED BY PACIFIC JOURNAL OF MATHEMATICS, A NON-PROFIT CORPORATION Printed at Jerusalem Academic Press, POB 2390, Jerusalem, Israel.

$$
\begin{gathered}
\text { Copyright (C) } 1975 \text { Pacific Journal of Mathematics } \\
\text { All Rights Reserved }
\end{gathered}
$$




\section{Pacific Journal of Mathematics}

\section{Vol. 58, No. $2 \quad$ April, 1975}

Zvi Artstein and John Allen Burns, Integration of compact set-valued functions . . . . . . . . . 297

Mark Benard, Characters and Schur indices of the unitary reflection group $[321]^{3} \ldots \ldots \ldots . .309$

Simeon M. Berman, A new characterization of characteristic functions of absolutely continuous

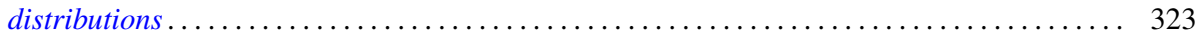

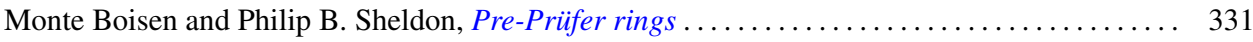

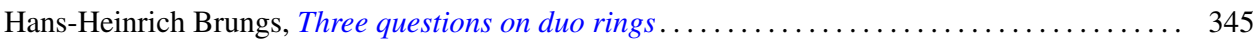

Iracema M. Bund, Birnbaum-Orlicz spaces of functions on groups................. 351

John D. Elwin and Donald R. Short, Branched immersions between 2-manifolds of higher

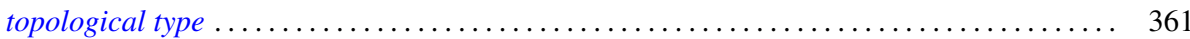

Eric Friedlander, Extension functions for rank 2, torsion free abelian groups . .......... 371

Jon Froemke and Robert Willis Quackenbush, The spectrum of an equational class of

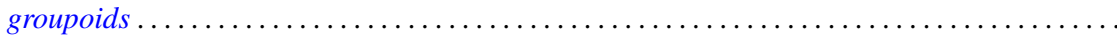

Barry J. Gardner, Radicals of supplementary semilattice sums of associative rings ...........

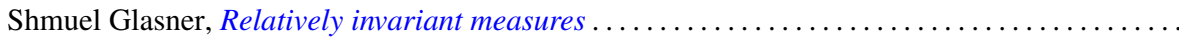

George Rudolph Gordh, Jr. and Sibe Mardesic, Characterizing local connectedness in inverse

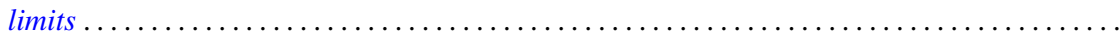

Siegfried Graf, On the existence of strong liftings in second countable topological spaces......

Stanley P. Gudder and D. Strawther, Orthogonally additive and orthogonally increasing

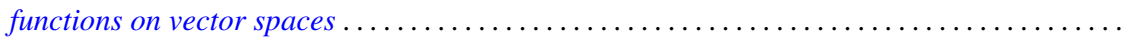

Darald Joe Hartfiel and Carlton James Maxson, A characterization of the maximal monoids and

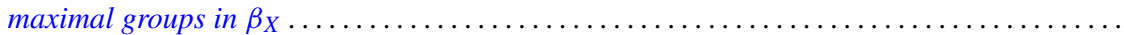

Robert E. Hartwig and S. Brent Morris, The universal flip matrix and the generalized faro-shuffle. .

William Emery Haver, Mappings between ANRs that are fine homotopy equivalences. .

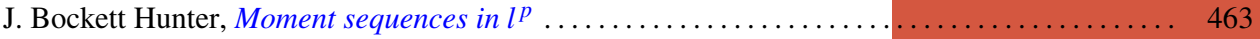

Barbara Jeffcott and William Thomas Spears, Semimodularity in the completion of a poset.... 467

Jerry Alan Johnson, A note on Banach spaces of Lipschitz functions . . . . . . . . . . . . 475

David W. Jonah and Bertram Manuel Schreiber, Transitive affine transformations on

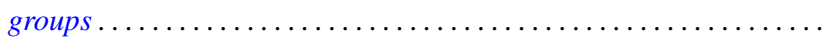

Karsten Juul, Some three-point subset properties connected with Menger's characterization of

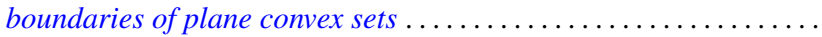

Ronald Brian Kirk, The Haar integral via non-standard analysis . . . . . . . . . . . . . 517

Justin Thomas Lloyd and William Smiley, On the group of permutations with countable

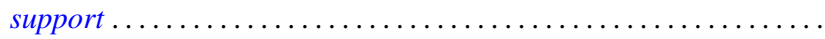

Erwin Lutwak, Dual mixed volumes .................................. 531

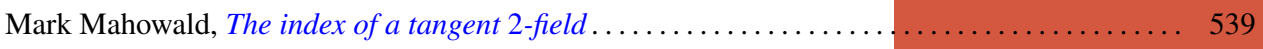

Keith Miller, Logarithmic convexity results for holomorphic semigroups . . . . . . . . . . . . 549

Paul Milnes, Extension of continuous functions on topological semigroups . . . . . . . . . . 553

Kenneth Clayton Pietz, Cauchy transforms and characteristic functions ................ 563

James Ted Rogers Jr., Whitney continua in the hyperspace $C(X) \ldots \ldots \ldots \ldots \ldots \ldots \ldots \ldots .569$

Jean-Marie G. Rolin, The inverse of a continuous additive functional . . . . . . . . . . . . 585

William Henry Ruckle, Absolutely divergent series and isomorphism of subspaces . ........ 605

Rolf Schneider, A measure of convexity for compact sets . ..................... 617

Alan Henry Schoenfeld, Continous measure-preserving maps onto Peano spaces .......... 627

V. Merriline Smith, Strongly superficial elements .......................... 643

Roger P. Ware, A note on quadratic forms over Pythagorean fields . . . . . . . . . . . . . . 651

Roger Allen Wiegand and Sylvia Wiegand, Finitely generated modules over Bezout rings . . . . 655

Martin Ziegler, A counterexample in the theory of definable automorphisms . . . . . . . . . 665 